\title{
A Comparative Study of the Biodegradation of the Surfactant Sodium Dodecyltriethoxy Sulphate by Four Detergent-degrading Bacteria
}

\author{
By STEPHEN G. HALES, ${ }^{\dagger}$ G. KEITH WATSON, 2 \\ KENNETH S. DODGSON ${ }^{1}$ AND GRAHAM F. WHITE ${ }^{1 *}$ \\ ${ }^{1}$ Department of Biochemistry, University College Cardiff, PO Box 78, Cardiff CF1 IXL, UK \\ 2 Unilever Research Port Sunlight Laboratory, Wirral, Merseyside L63 3JW, UK
}

(Received 12 August 1985; revised 14 November 1985)

\begin{abstract}
The ${ }^{35} \mathrm{~S}$-labelled metabolites produced during biodegradation of sodium dodecyltriethoxy $\left[{ }^{35}\right.$ S $]$ sulphate (SDTES) by four bacterial isolates were identified and quantified. All four isolates used ether-cleavage as the predominant primary degradation pathway. In two of the organisms, the etherase system (responsible for approx. $60-70 \%$ of primary biodegradation) liberated mono-, di- and triethylene glycol monosulphates in substantial proportions, the last two esters undergoing some further oxidation to acetic acid 2-(ethoxy sulphate) and acetic acid 2-(diethoxy sulphate), respectively. For these isolates, liberation of $\mathrm{SO}_{4}^{2-}$ directly from SDTES was also significant $(30-40 \%)$ and the organisms were shown to contain alkylsulphatases active towards SDTES. For the remaining two isolates, etherase action was even more important (responsible for $>80 \%$ of primary biodegradation) and was restricted almost totally to the alkyl-ether bond to generate mainly triethylene glycol sulphate, some of which was further oxidized. Very small amounts of diethylene glycol monosulphate were also produced, but its mono-homologue, and the oxidation products of both these esters, were absent. Small amounts of inorganic sulphate (approx. 10\%) were liberated by these isolates and one of them also produced compounds tentatively identified as intermediates of $\omega$-/ $\beta$-oxidation.
\end{abstract}

\section{INTRODUCTION}

Alkylethoxy sulphates are important components of certain detergent formulations such as hair shampoos, combining good foaming properties with low skin irritancy (Shore \& Berger, 1976). A typical component is sodium dodecyltriethoxy sulphate (SDTES; see Table 1 for structures and abbreviations), and possible routes for its biodegradation are shown in Fig. 1. Although primary biodegradation of linear alkylethoxy sulphate surfactants in mixed cultures is rapid and complete (Swisher, 1970; Yoshimura \& Masuda, 1982), there is evidence that mineralization of the ethoxylate moiety is slower than that of the alkyl chain (Vashon \& Schwab, 1982). Support for this view has come from work in our Cardiff laboratory (Hales et al., 1982), which has shown that residues from the ethoxy sulphate moiety accumulate in the medium during biodegradation of SDTES by a sewage isolate, Pseudomonas sp. strain DES1. The initial ether-cleavage occurred at each of the three ether bonds in the SDTES molecule, to produce mono-, di- and triethylene glycol monosulphates in the proportions $20: 45: 35$. The latter two compounds were subsequently oxidized to acetic acid 2-(ethoxy sulphate) and acetic acid 2(diethoxy sulphate) respectively (see Table 1). The removal of $\mathrm{SO}_{4}^{2-}$ directly from SDTES was a minor contribution to primary biodegradation but its importance increased when SDTES was present as a component of a commercial detergent mixture, as would normally be the case in the environment. A third possible route for degradation of SDTES is $\omega$-oxidation followed by $\beta$ -

† Present address: Unilever Research Port Sunlight Laboratory, Wirral, Merseyside L63 3JW, UK.

Ahbreviations: see Table 1

$0001-2889 \circlearrowleft 1986$ SGM 


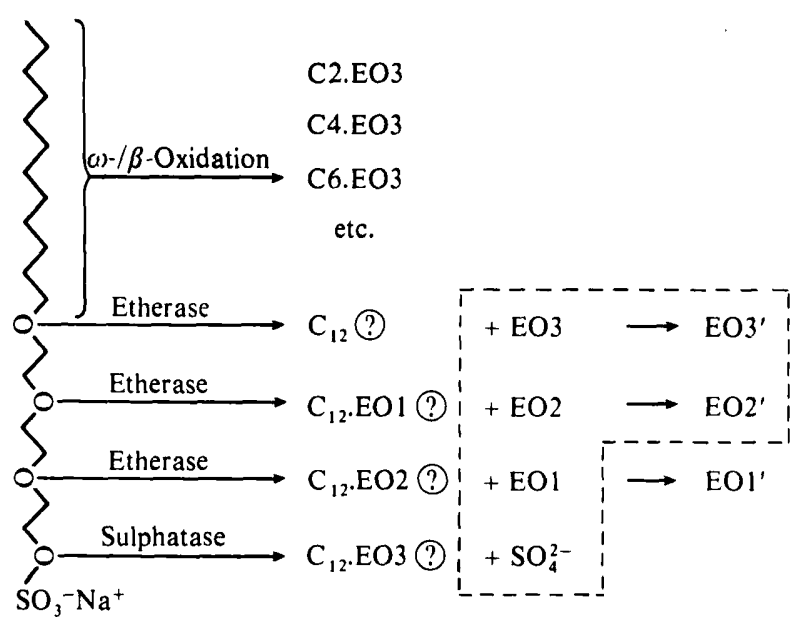

Fig. 1. Possible routes for biodegradation of SDTES. See Table I for key to abbreviations and chemical structures. () indicates that the terminal group of the immediate product of etherase action may be alcohol, aldehyde or carboxylic acid. Metabolites bounded by the dotted line are known to occur during biodegradation of SDTES by Pseudomonas sp. strain DESI. Other metabolites have not been identified hitherto.

Table 1. Formulae, names and abbreviations of surfactant and its known and potential metabolic products

Metabolic pathways leading to the formation of these compounds are summarized in Fig. 1, and further details are in the text. The formulae and names are arranged to show the structural relationships in terms of homologous groups of the alcoholic $(b)$ and acidic $(c)$ products of etherase action, and $\omega$ oxidation products $(d)$ derived from the parent surfactant $(a)$.

(a) Parent compound

Sodium dodecyl

triethoxy sulphate (SDTES)

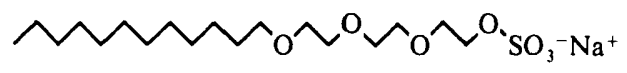

(b) Alcoholic products

Triethylene glycol monosulphate (EO3)

Diethylene glycol monosulphate (EO2)

Monoethylene glycol monosulphate (EOI)<smiles>O=S(=O)([O-])OCCOCCOCCO</smiles><smiles>O=S(=O)([O-])OCCOCCO</smiles><smiles>O=S(=O)([O-])OCCO</smiles>

(c) Acidic products

Acetic acid

2-(diethoxy sulphate) (EO3')

Acetic acid

2-(ethoxy sulphate)

(EO2')

Acetic acid

2-sulphate (EO1')
${ }^{\mathrm{HOOC}}{ }^{\mathrm{O}} \mathrm{CO}{ }^{\mathrm{O}} \mathrm{SO}_{3}^{-}$<smiles>O=C(O)COCCOS(=O)(=O)O</smiles>

${ }^{\mathrm{HOOC}}{ }^{\mathrm{O}} \mathrm{SSO}_{3}-$

(d) $\omega$-Oxidation products

Acetic acid 2-(triethoxy sulphate) (C2.EO3)

Butyric acid 4-(triethoxy sulphate) (C4.EO3)

Hexanoic acid 6-(triethoxy sulphate) (C6.EO3)

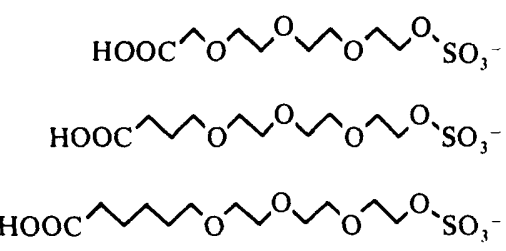

oxidation, a pathway known to occur for example in the microbial degradation of alkylbenzene sulphonate surfactants (Cain, 1981) and in the mammalian degradation of alkyl sulphates (Denner et al., 1969; Maggs et al., 1982) and alkylethoxy sulphates (Taylor et al., 1978). However, this pathway was not observed during studies on the degradation of SDTES by Pseudomonas sp. DESI. 
The present study was undertaken to extend the earlier work with Pseudomonas sp. DES1 by including other isolates chosen for their known abilities to metabolize simple alkyl sulphate surfactants, non-ionic alkyl ethoxylate surfactants or polyethylene glycols. The relative contributions of the three main routes of primary biodegradation (etherase, sulphatase and $\omega-/ \beta$ oxidation, see Fig. 1) were assessed and the results confirmed that while the etherase route is quantitatively the most important in the bacterial degradation of SDTES, the other routes may also make significant contributions. The work also provided useful indications of the types of intermediate that might accumulate during primary biodegradation of SDTES in the environment.'

\section{METHODS}

Chemicals. Except for items listed below, all reagents were of Analar grade, purchased from BDH. Commercial alkyl polyethoxy sulphate surfactants, Empicol ESB/3S and Dobanol 25/3EO sulphate (i.e. sulphated Dobanol $25 / 3 \mathrm{EO}$ ), were supplied by Albright \& Wilson. Empicol ESB/3S contained mainly $C_{12}$ alkyl chains and mainly 1 to 4 (but up to 10) ethylene glycol units per molecule. Dobanol 25/3EO sulphate contained $C_{12}$ to $C_{15}$ alkyl chains ( $25 \%$ of which carried 2-methyl or 2-ethyl branches), and mainly 1 to 4 ethylene glycol units per molecule. Both preparations also contained 5-10\% linear alkyl sulphates. Sources of unlabelled SDTES and [dodecyl-1${ }^{14} \mathrm{C}$ SSDTES, and methods for synthesizing glycol monosulphates and oxidized glycol sulphates have been described previously (Hales et al., 1982). Dodecyltriethoxy alcohol (dodecyltriethoxylate) was obtained from the Nikko Chemical Company.

$\left[{ }^{35} S\right]$ SDTES. Crude $\left[{ }^{35}\right.$ S]SDTES was prepared as described previously (Hales et al., 1982), and purified by preparative TLC on silica gel G-60 (0.5 mm layer; Merck). After development in propan-2-ol/chloroform/ methanol/water $\left(50: 20: 5: 2 \cdot 4\right.$, by vol.) the major band $\left(R_{F} 0.5\right)$ was scraped from the glass plate and [ $\left.{ }^{35} S\right]$ SDTES, now free from inorganic sulphate, was eluted with repeated water washings (10-20 times) until the recovery was $>95 \%$. The partially purified $\left[{ }^{35}\right.$ S $]$ SDTES eluate was freeze-dried, dissolved in the minimum volume of ethanol and applied to a second preparative TLC plate, which was developed with toluene/acetone $(4: 1, v / v)$ to separate ${ }^{3}$ S S]SDTES from non-polar contaminants. The [35S]SDTES was eluted with water as before and the resulting solution was freeze-dried. The residue was redissolved in $2 \mathrm{ml}$ water and stored frozen. The radiochemical purity of the final product was $>99 \%$ based on TLC analysis, its specific activity was $1 \mathrm{MBq} \mathrm{g}^{-1}$, and the yield was $14 \%$.

Isolation of organisms and culture conditions. Pseudomonas sp. strain DES1 was isolated from the effluent of an activated sludge test plant at Unilever Research Port Sunlight Laboratory, UK (Hales et al., 1982).

Pseudomonas sp. strain C12B was isolated from soil near a sewage outfall in Athens, Georgia, USA, by selection with SDS as sole source of carbon and energy (Payne \& Feisal, 1963).

Pseudomonas sp. strain SC25A was purified at Unilever Research Port Sunlight Laboratory from a mixed sewage culture that had been adapted to grow on a mixture of polyethylene glycols. The organism could also degrade the alcohol ethoxylate surfactant Dobanol 25/7.

The fourth organism studied (strain TES5) was isolated from a culture medium containing Empicol ESB/3S that had been inoculated with a strain then known as TEG5, an organism able to grow on triethylene glycol (Fincher \& Payne, 1962).

All four organisms were maintained by monthly transfers on Oxoid Agar (no. 3) made up in a mineral salts medium (Hales et al., 1982) and containing $0.5 \%$ Empicol ESB/3S as the sole source of carbon. Inocula were prepared by growing each organism in $250 \mathrm{ml}$ Erlenmeyer flasks containing $25 \mathrm{ml} 0.063 \%$ (w/v) Empicol ESB/3S in mineral salts ( $\mathrm{pH} 7$ ), to mid-exponential phase (16 h for Pseudomonas spp. strains DES1, C12B and SC25A; $45 \mathrm{~h}$ for strain TES5). Samples (1 ml) of these cultures were used to inoculate $100 \mathrm{ml}$ Erlenmeyer flasks containing $10 \mathrm{ml}$ $0.063 \%(\mathrm{w} / \mathrm{v})$ Empicol ESB/3S in the mineral salts medium supplemented with $\left[{ }^{35} S\right]$ SDTES (37 kBq per flask). Flasks were shaken at 100 r.p.m, for up to $350 \mathrm{~h}$ at $30^{\circ} \mathrm{C}$. Samples (approx. $0.1 \mathrm{ml}$ ) were removed aseptically at intervals, and aliquots $(10 \mu \mathrm{l})$ analysed by two-dimensional TLC.

TLC of metabolites. ${ }^{35} \mathrm{~S}$-Labelled metabolites produced during the biodegradation of SDTES were separated and identified by two-dimensional TLC on cellulose plates (Eastman Kodak) as described previously (Hales et al., 1982). Plates spotted with $10 \mu \mathrm{l}$ aliquots of whole medium were developed in the first dimension $(5 \mathrm{~cm})$ with propan-1-ol/ammonia (sp. gr. 0.880$)(7: 3, \mathrm{v} / \mathrm{v})$ or propan-2-ol/water $(7: 3, \mathrm{v} / \mathrm{v})$ and, after overnight drying, in the second dimension $(8.5 \mathrm{~cm})$ with isobutyric acid $/ 0.5 \mathrm{M}$-aqueous ammonia $(5: 3, \mathrm{v} / \mathrm{v})$. Radioactive areas on developed chromatograms were located by autoradiography with Kodak X-omat RPI X-ray film and the amount of ${ }^{35} \mathrm{~S}$-labelled material in each area was assayed as described previously (Hales et al., 1982).

${ }^{1+} \mathrm{C}$-Labelled metabolites produced by strain TES 5 grown on $\left[{ }^{+} \mathrm{C}\right]$ SDTES were also separated by TLC. For twodimensional TLC, each sample was applied to the corner of a cellulose plate $(6.6 \times 10 \mathrm{~cm})$ and developed in propan-2-ol/water $(7: 3, \mathrm{v} / \mathrm{v})$ or propan-2-ol/ammonia (sp. gr. 0.880$)(7: 3, \mathrm{v} / \mathrm{v})$ in the first dimension, followed by isobutyric acid $/ 0.5 \mathrm{M}$-aqueous ammonia $(5: 3, \mathrm{v} / \mathrm{v})$ in the second dimension. For one-dimensional TLC, samples were applied to cellulose plates and developed in either chloroform/methanol/water $(8: 5: 1$, by vol.) or isobutyric 
acid/0.5 M-aqueous ammonia $(5: 3, \mathrm{v} / \mathrm{v})$. Alternatively, silica gel G-60 plates were used and the developing solvent was either propan-2-ol/water $(7: 3, \mathrm{v} / \mathrm{v})$, or propan-2-ol/ammonia (sp. gr. $0.880(7: 3, \mathrm{v} / \mathrm{v})$, or propan-2$\mathrm{ol} /$ chloroform/methanol/water $(40: 20: 5: 2 \cdot 4$, by vol. $)$.

Gel zymography of sulphatases in cell extracts. Each of the four isolates was grown in $50 \mathrm{ml}$ sterile medium containing $0.063 \%(\mathrm{w} / \mathrm{v})$ Empicol ESB/3S in mineral salts medium, in $250 \mathrm{ml}$ Erlenmeyer flasks. Cultures were incubated with shaking, for $16 \mathrm{~h}$ at $30^{\circ} \mathrm{C}$. Cells were harvested by centrifugation $\left(9500 \mathrm{gav}_{\mathrm{a}}, 15 \mathrm{~min}\right)$ and extracts prepared for electrophoresis as described by White et al. (1984). Polyacrylamide gel electrophoresis was done by the method of Payne \& Painter (1971) as modified by Dodgson et al. (1974), except that the gels contained 5.5\% $(w / v)$ polyacrylamide. Extruded gels were stained for primary alkylsulphatases by incubation with SDS; enzyme bands were revealed by the appearance of insoluble droplets of dodecanol at the site of liberation. For secondary alkylsulphatases, the staining solution contained a mixture of secondary decyl sulphates (mainly the 2- and 5isomers: see Dodgson et al., 1974; Matcham \& Dodgson, 1977). Sulphatase activity towards SDTES was detected by incubating gels at $30^{\circ} \mathrm{C}$ in a solution containing $10 \mathrm{~mm}$-SDTES and $20 \mathrm{~mm}-\mathrm{BaCl}_{2}$ in $20 \mathrm{~mm}-\mathrm{Tris} / \mathrm{HCl}, \mathrm{pH} \mathrm{7.8}$. Although the liberated alcohol was not sufficiently insoluble to form a visible precipitate, white bands were formed because the presence of $\mathrm{Ba}^{2+}$ ions led to precipitation of liberated $\mathrm{SO}_{4}^{2-}$.

\section{RESULTS AND DISCUSSION}

Four organisms (strain TES5 and the Pseudomonas spp. strains DES1, C12B and SC25A) were grown on the commercial alkylethoxy sulphate surfactant Empicol ESB/3S supplemented with [ ${ }^{35}$ S]SDTES. The patterns of ${ }^{35}$ S-labelled metabolites were determined by two-dimensional TLC (Table 2). The pattern for Pseudomonas sp. DES1 has been detailed elsewhere (Hales et al., 1982), but is included in Table 2 for comparison with other isolates.

\section{Metabolites produced by Pseudomonas sp. strain C12B}

Originally isolated for its ability to degrade alkyl sulphate surfactants, this organism grew well on Empicol ESB/3S supplemented with $\left[{ }^{35}\right.$ S]SDTES. Growth reached stationary phase within $24 \mathrm{~h}$ and the foaming had almost disappeared. Biodegradation of $\left[{ }^{35}\right.$ S]SDTES began in the $24 \mathrm{~h}$ growth period but it was slow and still incomplete after $342 \mathrm{~h}$. All three glycol monosulphates (EO1, EO2 and EO3) and inorganic sulphate were formed in substantial amounts, together with smaller amounts of the oxidation products EO2' and EO3' (Table 1). However, EO1' and $\omega-/ \beta$ oxidation products were absent. The proportions of inorganic $\left[{ }^{35} S\right]$ sulphate (arising from sulphatase attack) and total organic ${ }^{35} \mathrm{~S}$-labelled metabolites (arising from etherase attack) were in the approx. ratio 1:2 in all three samples (Table 3); this is very similar to that observed for Pseudomonas sp. DES1 (Hales et al., 1982). When the organic metabolites listed in Table 2 were grouped according to whether they contained either one, two or three ethylene glycol units, the relative amounts of ${ }^{35} \mathrm{~S}$ label in each group indicated the extent of cleavage at each ether bond. In Pseudomonas sp. $\mathrm{C} 12 \mathrm{~B}$ the three ether bonds (in order from the alkyl-ether bond) were cleaved in the average proportions $25: 38: 37$. Again the pattern was similar to that in Pseudomonas sp. DES1, but the glycol sulphates were oxidized more slowly in Pseudomonas sp. C12B.

\section{Metabolites produced by Pseudomonas sp. strain SC25A}

Like Pseudomonas sp. DES1, this bacterium grew well on Empicol ESB/3S, reaching stationary phase within $24 \mathrm{~h}$ by which time all foaming had disappeared. However, the pattern of metabolite production was quite different from that of Pseudomonas sp. DESI and C12B. The large amounts of EO3 produced initially were subsequently oxidized extensively (Table 2). Lesser amounts of EO2 and inorganic sulphate were also observed but EO1, EO1' and EO2' were all absent. The ratio of inorganic sulphate to sulphated organic metabolites was $1: 9$ (Table 3), showing that, for this organism, etherase-cleavage was the dominant route. If it is assumed, as above, that the proportions of organic sulphate esters produced were the same as the proportions of ether bonds cleaved, then the three ether bonds (in order from the alkyl-ether bond) were broken in the ratio $95: 5: 0$. Clearly, primary biodegradation of SDTES in this organism was restricted not only to the predominant ether-cleavage route but also specifically to the cleavage of the alkyl-ether bond. 
Table 2. Relative amounts of residual $\left[{ }^{35} S\right] S D T E S$ and its ${ }^{35} S$-labelled metabolites during biodegradation of Empicol ESB/3S by different bacterial isolates

\begin{tabular}{|c|c|c|c|c|c|c|c|c|c|c|}
\hline \multirow[b]{2}{*}{ Isolate } & \multirow{2}{*}{$\begin{array}{l}\text { Culture } \\
\text { age (h) }\end{array}$} & \multicolumn{9}{|c|}{ Relative amounts of $\left[{ }^{35}\right.$ S]SDTES and its ${ }^{35}$ S-labelled metabolites* } \\
\hline & & SDTES & EO3 & EO2 & EOI & $\mathrm{EO3}^{\prime}$ & $\mathrm{EO} 2^{\prime}$ & $\mathrm{EOl}^{\prime}$ & $\mathrm{SO}_{4}^{2-}$ & $\omega / \beta$ \\
\hline \multirow[t]{3}{*}{ DESI $\dagger$} & 14 & 74 & 3 & 4 & 3 & 1 & 1 & 0 & 14 & 0 \\
\hline & 120 & 0 & 7 & 25 & 23 & 5 & 6 & 0 & 34 & 0 \\
\hline & 270 & 0 & 0 & 0 & 23 & 12 & 30 & 0 & 35 & 0 \\
\hline \multirow[t]{3}{*}{$\mathrm{C} 12 \mathrm{~B}$} & 72 & 50 & 9 & 13 & 11 & 0 & 0 & 0 & 18 & 0 \\
\hline & 147 & 17 & 10 & 18 & 23 & 2 & 2 & 0 & 27 & 0 \\
\hline & 342 & 5 & 12 & 21 & 21 & 4 & 2 & 0 & 36 & 0 \\
\hline \multirow[t]{2}{*}{ SC25A } & 9 & 0 & 56 & 4 & 0 & 29 & 0 & 0 & 11 & 0 \\
\hline & 147 & 0 & 13 & 5 & 0 & 73 & 0 & 0 & 9 & 0 \\
\hline TES5 & 294 & 0 & 72 & 1 & 0 & 11 & 0 & 0 & 6 & 11 \\
\hline
\end{tabular}

* Expressed as a percentage of total ${ }^{35} \mathrm{~S}$-labelled material (SDTES and its metabolites) present in the incubation mixture. See text for experimental details. See Table 1 for key to abbreviations; $\omega / \beta$, products of $\omega$ - $/ \beta$-oxidation.

$\dagger$ Data for strain DESI taken from Hales et al. (1982)

Table 3. Relative amounts of ${ }^{35} S$-labelled metabolites arising from etherase, sulphatase and $\omega$-/ $\beta$-oxidation pathways during biodegradation of Empicol ESB/3S by different bacterial isolates

\begin{tabular}{lcccc}
\multirow{2}{*}{ Isolate } & $\begin{array}{c}\text { Culture } \\
\text { age (h) }\end{array}$ & Etheraset & Sulphatase & $\omega$-/ $\beta$-Oxidation \\
DESI & 14 & 43 & 57 & 0 \\
& 120 & 66 & 34 & 0 \\
C12B & 270 & 65 & 35 & 0 \\
& 72 & 65 & 35 & 0 \\
SC25A & 147 & 67 & 33 & 0 \\
& 342 & 61 & 39 & 0 \\
TES5 & 9 & 89 & 11 & 0 \\
& 147 & 91 & 9 & 11
\end{tabular}

\footnotetext{
* Expressed as percentages of total ${ }^{35} \mathrm{~S}$-labelled metabolites (i.e. excluding residual SDTES).

† Combined amounts of mono-, di- and triethylene glycol monosulphates and their immediate oxidation products. Data derived from Table 2.
}

\section{Metabolites produced by strain TES5}

This organism grew on SDTES alone, reaching stationary phase in $5 \mathrm{~d}$, which is faster than Pseudomonas sp. DES1. Strain TES5 also grew on Empicol ESB/3S in liquid culture at a rate similar to that of the other organisms, and removed all foaming within $48 \mathrm{~h}$. Biodegradation of $\left[{ }^{35}\right.$ S]SDTES resulted in the formation of large amounts of EO3 and much smaller amounts of EO3', EO2 and inorganic sulphate (Table 2). The ratio of sulphatase/etherase products was $1: 14$ (Table 3; cf. Pseudomonas sp. SC25A), and the three ether bonds (in order from the alkyl-ether bond) were cleaved in the ratio $99: 1: 0$. Obviously the etherase system in this organism represented the dominant pathway and it, too, was highly specific for the alkyl-ether linkage.

In addition to the foregoing, there was a group of metabolites present $(11 \%$ of the total, three or sometimes four spots) which had not been encountered hitherto; they ran close together on two-dimensional TLC systems in a position between the oxidized glycol monosulphates and SDTES. When strain TES5 was grown in a medium supplemented with $\left[1-{ }^{14} \mathrm{C}\right]$ SDTES instead of the ${ }^{35} \mathrm{~S}$-labelled SDTES, the same intermediates were detected. Therefore, the metabolites must have contained both the ${ }^{35} \mathrm{~S}$ label of the sulphate moiety and the ${ }^{14} \mathrm{C}$ label at $\mathrm{C}-1$ of the alkyl chain, so they could not have arisen from the actions of either sulphatase or etherase 


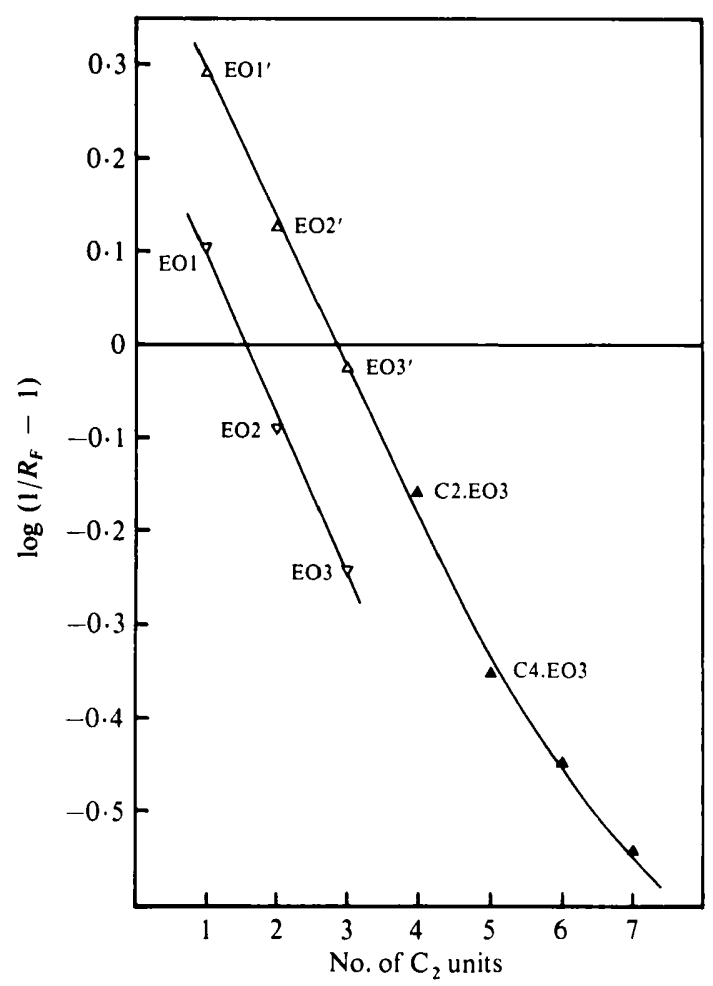

Fig. 2. The Martin relationship for homologous series of glycol monosulphates, oxidized glycol monosulphates and novel intermediates of $\left[{ }^{35}\right.$ S]SDTES degradation produced by strain TES5. Experimental details are given in the text and compound name abbreviations in Table $1 . \nabla$, Glycol monosulphates (standard compounds); $\triangle$, oxidized glycol monosulphates (standard compounds) $; \boldsymbol{\Delta}$, metabolites of $\left[{ }^{35} S\right]$ SDTES produced by strain TES5, tentatively identified as $\omega$-/ $\beta$-oxidation intermediates (see text).

enzymes. The possibility then remained that these metabolites arose from $\omega$-/ $\beta$-oxidation of the dodecyl chain of SDTES.

When samples of medium containing the ${ }^{14} \mathrm{C}$-labelled metabolites were analysed by silica gel G-60 TLC in propan-2-ol/water $(7: 3, \mathrm{v} / \mathrm{v})$, the labelled metabolites co-migrated as a single spot with an $R_{F}$ value very similar to that of oxidized glycol monosulphates $(0.64)$ but less than that of the glycol monosulphates $(0 \cdot 80)$. In this solvent system, glycol sulphates and their oxidized derivatives migrated according to the nature of the non-sulphated terminal functional group and not according to molecular size (Hales et al., 1982). Thus it appeared that the ${ }^{14} \mathrm{C}$-labelled metabolites contained carboxylic acid terminal groups, in keeping with an $\omega$-/ $\beta$-oxidation pathway.

The glycol sulphates (EO1, EO2 and EO3) and their oxidized counterparts constitute separate homologous series based on the $\mathrm{OCH}_{2} \mathrm{CH}_{2}$ structural unit. Such series should conform to the Martin relationship, which states that values of $\log \left(1 / R_{F}-1\right)$ are linear functions of the number of structural units, provided that the $R_{F}$ values are in the range $0 \cdot 1$ to $0 \cdot 7$ (Brenner et al., 1962). In the present study this was achieved on cellulose plates with isobutyric acid/0.5 M-ammonia $(7: 3$, $\mathrm{v} / \mathrm{v}$ ) as solvent system. The corresponding plots for the glycol sulphates and oxidized sulphates based on the number of $\mathrm{C}_{2}$ units are shown in Fig. 2. $\omega$-/ $\beta$-Oxidation of SDTES would also give rise to the homologous series $-\mathrm{OSO}_{2}\left(\mathrm{OCH}_{2} \mathrm{CH}_{2}\right)_{3} \cdot \mathrm{O}\left(\mathrm{CH}_{2} \mathrm{CH}_{2}\right)_{n} \cdot \mathrm{CH}_{2} \mathrm{COOH}$. The ultimate product of $\beta$-oxidation $(n=0 ; \mathrm{C} 2$.EO3) is in fact homologous with the oxidized glycol monosulphates (see Table 1). The value of $\log \left(1 / R_{F}-1\right)$ for the end-product of $\beta$-oxidation should therefore fall on the Martin plot for the oxidized glycol monosulphates at the point 
corresponding to four $\mathrm{C}_{2}$ units, and this was indeed so (Fig. 2). The metabolite with the next lowest value of $\left(1 / R_{F}-1\right)$ also closely fitted the linear plot and was tentatively identified as the homologue with $n=1$ (C4.EO3, see Table 1). For the other metabolites the plot diverged from linearity, presumably because the homologous structural unit had changed from $\mathrm{OCH}_{2} . \mathrm{CH}_{2}$ to $\mathrm{CH}_{2} \cdot \mathrm{CH}_{2}$. Collectively these results suggest the existence in strain TES5 of an $\omega$ - (or possibly a subterminal-) oxidation system followed by $\beta$-oxidation of the alkyl chain of SDTES to remove $\mathrm{C}_{2}$ units. The contribution of this pathway to SDTES degradation in strain TES5 is not insignificant, accounting for $11 \%$ of the total biodegradation, almost twice as much as the sulphatase route.

\section{Alkylsulphatases in cell extracts}

Primary biodegradation of SDTES in some pure cultures proceeded with liberation of considerable quantities of inorganic sulphate which, certainly for Pseudomonas sp. DES1 (Hales et al., 1982) and probably also for the other strains, arose directly from the surfactant. In addition, Empicol ESB/3S contains simple alkyl sulphates (e.g. dodecyl sulphate), which are biodegradable by alkylsulphatases, like those present in Pseudomonas sp. C12B (Dodgson \& White, 1983; Dodgson et al., 1982). SDTES-degrading organisms were examined therefore by gel zymography for enzymes that could desulphate SDTES. Pseudomonas sp. DES1 grown on Empicol ESB/3S always produced two primary enzyme bands (designated DP1 and DP2) and two secondary enzymes (DS1 and DS2). Up to three additional secondary bands with mobilities intermediate between DS1 and DS2 were observed under various conditions. The enzymes DP2 and DS2, which were both induced by growth on Empicol ESB/3S, SDTES or SDS, were always present together, and possessed very similar electrophoretic mobilities. Moreover, the DP2/DS2 band was also coincident with a band that liberated sulphate from SDTES. The mobilities of the three enzyme bands were altered to the same extent when the polyacrylamide concentration in gels was raised to $6.8 \%(\mathrm{w} / \mathrm{v})$. Collectively, these results suggested that a single protein was responsible for the three activities observed.

Pseudomonas sp. C12B can produce two primary alkylsulphatases, P1 (constitutive) and P2 (inducible), together with three secondary enzymes, S1, S2 and S3 (Dodgson \& White, 1983; Dodgson et al., 1982). Cells grown on Empicol ESB/3S yielded cell extracts containing P1 and the substrate-inducible P2 enzyme active against SDS. The S1 and traces of the S2 alkylsulphatase were also present, but not S3. On some occasions, gels incubated with SDTES and $\mathrm{BaCl}_{2}$ contained a faint, transient band of precipitated $\mathrm{BaSO}_{4}$ in a position corresponding to the $\mathrm{P} 2$ enzyme. On other occasions, the band did not appear at all even though $\mathrm{P} 2$ was present, suggesting that the latter enzyme may not be responsible for desulphation of SDTES. Previous studies (Cloves et al., 1980) showed that SDTES was a relatively weak inducer for the P2 enzyme compared with SDS, and it was probable that, in the present study, simple alkyl sulphates (e.g. SDS) present in Empicol ESB/3S were mainly responsible for induction of the P2 enzyme.

Gel zymograms for Pseudomonas sp. SC25A closely resembled those for Pseudomonas sp. DES1 grown under similar conditions. However, electrophoresis of a mixture of extracts of the two strains revealed slight differences in the mobilities of the primary enzymes.

In view of the minor contribution of sulphatase action $(6 \%$ of the total) to biodegradation of SDTES in strain TES5, no attempt was made to detect SDTES sulphatase in extracts of these cells.

\section{CONCLUSIONS}

Studies with four different naturally-occurring organisms have shown that the three possible routes for primary biodegradation of alkylethoxy sulphate surfactants (etherase, sulphatase and $\omega$-/ $\beta$-oxidation; see Fig. 1) can all operate in bacteria. The main route in all four isolates was ether-cleavage (Table 2). In two of the isolates (Pseudomonas spp. DES1 and C12B), the etherase system was non-specific, cleaving each of the three ether bonds of SDTES in substantial proportions. In the other two organisms (strains SC25A and TES5) specificity was much higher, and cleavage occurred almost entirely at the alkyl-ether linkage. All organisms could produce 
triethylene glycol monosulphate (EO3) and its oxidation product acetic acid 2-(diethoxy sulphate) (EO3'). Diethylene glycol monosulphate (EO2) was also produced by all isolates but only the most prolific producers (strains DES1 and C12B) generated detectable amounts of its oxidation product, acetic acid 2-(ethoxy sulphate) (EO2'). These same two strains were also alone in liberating monoethylene glycol monosulphate (EO1), and none of the four strains produced detectable amounts of acetic acid 2-sulphate (EO1').

Sulphatase-cleavage also made a significant contribution to primary biodegradation, especially in those isolates with ether-cleaving systems of low specificity (strains DES1 and $\mathrm{C} 12 \mathrm{~B})$. In the three organisms tested, multiple primary and secondary alkylsulphatases were observed, a feature held in common with a number of other alkyl sulphate degrading bacteria (Dodgson et al., 1982; White et al., 1984).

Perhaps the most unexpected result in the present study was the detection of intermediates tentatively identified as products of an $\omega$-/ $\beta$-oxidation (strain TES5, Table 2 ). Alternatively these intermediates could have been produced by subterminal oxidation and cleavage between $C-11$ and $C-10$, or C-9 and C-8 of the dodecyl chain, followed by varying amounts of $\beta$-oxidation. Although the contribution of this pathway was small ( $11 \%$ of the total), that it was involved at all was surprising because the route appears normally to be used only when other mechanisms are unavailable, e.g. bacterial degradation of alkylbenzene sulphonates (Cain, 1981) and alkanes (Ratledge, 1978) or mammalian degradation of alkyl sulphates (Denner et al., 1969; Maggs et al., 1982) and alkylethoxy sulphates (Taylor et al., 1978).

Recently, combinations of $\omega$-/ $\beta$-oxidation and ether-cleavage have also been proposed to account for the metabolite patterns observed during degradation of non-ionic alkyl ethoxylates by mixed sewage cultures, but whether or not the different pathways were present in single species was not established (Steber \& Wierich, 1985). In the present study, it is clear that in three of the organisms tested, two of the possible mechanisms operated simultaneously, and in the fourth organism all three mechanisms were involved simultaneously in the degradation of SDTES. The ether-cleavage route was always dominant and this is advantageous to the organisms for two reasons. First, although sulphatase-cleavage is well established as the initiating step in the degradation of primary and secondary alkyl sulphate surfactants, desulphation of SDTES produces dodecyltriethoxy alcohol (dodecyl triethoxylate) which still must undergo ether-cleavage to render the dodecyl chain available for assimilation (Ichikawa $e t$ al., 1978). Second, although $\omega$ - $/ \beta$-oxidation results in the immediate assimilation of $C_{2}$ units, it is an inefficient use of the alkyl chain because $\beta$-oxidation becomes progressively more difficult as the site of cleavage approaches the hydrophilic moiety. In contrast, ether-cleavage at the alkylether linkage liberates a dodecyl chain that can be completely assimilated by $\beta$-oxidation.

Clearly the etherase and sulphatase pathways for biodegradation of SDTES are widely distributed. Moreover they may also be accompanied by an $\omega$ - $/ \beta$-oxidation route. Combination of the three routes in pure cultures led to accumulation of glycols, glycol sulphates, oxidized glycol sulphates and short-chain alkanoic acids, $\omega$-substituted with triethylene glycol monosulphate. Formation of some or all of these compounds might therefore be anticipated during biodegradation of alkylethoxy sulphate surfactants in environmentally relevant situations like sewage treatment plants or river-water. The following paper describes the formation of such materials from SDTES and their degree of persistence in such circumstances.

The award of a CASE Studentship Award to S.G.H. from the Science and Engineering Research Council is gratefully acknowledged.

We thank Dr N. J. Russell for reading and commenting on the manuscript.

\section{REFERENCES}

Brenner, M., Viederweisser, A., Pataki, G. \& WEBBER, R. (1962). Theoretical aspects of thin-layer chromatography. In Thin-Layer Chromatography, A Laboratory Handbook, pp. 75-133. Edited by E. Stahl. Berlin: Springer-Verlag.

CaIN, R. B. (1981). Microbial degradation of surfac- tants and builder components. In Microbial Degradation of Xenobiotics and Recalcitrant Compounds. FEMS Symposium no. 12, pp. 325-370. Edited by T. Leisinger, R. Hutter, A. M. Cook \& J. Niiesch. London: Academic Press.

Cloves, J. M., Dodgson, K. S., White, G. F. \& 
FitzGerald, J. W. (1980). Specificity of P2 primary alkylsulphohydrolase induction in the detergentdegrading bacterium Pseudomonas C12B. Biochemical Journal 185, 13-21.

Denner, W. H. B., Olavesen, A. H., Powell, G. M. \& Dodgson, K. S. (1969). The metabolism of potassium dodecyl $\left[{ }^{35}\right.$ S $]$ sulphate in the rat. Biochemical Journal 111, 43-51.

Dodgson, K. S. \& WhITE, G. F. (1983). Some microbial enzymes involved in the biodegradation of sulphated surfactants. In Topics in Enzyme and Fermentation Biotechnology, vol. 7, pp. 90-155. Edited by A. Wiseman. Chichester: Ellis-Horwood. Dodgson, K. S., Fitzgerald, J. W. \& Payne, W. J. (1974). Chemically defined inducers of alkylsulphatases present in Pseudomonas C12B. Biochemical Journal 138, 53-62.

Dodgson, K. S., White, G. F. \& Fitzgerald, J. W. (1982). The alkylsulfatases. In Sulfatases of Microbial Origin, vol. 1, pp. 9 48. Boca Raton: CRC Press.

FInCHER, E. L. \& PAYNe, W. J. (1962). Bacterial utilisation of ether glycols. Applied Microbiology 10 , 542-547.

Hales, S. G., Dodgson, K. S., White, G. F., Jones, N. \& WATSON, G. K. (1982). Initial stages in the biodegradation of the surfactant sodium dodecyltriethoxy sulphate by Pseudomonas sp. strain DES1. Applied and Entironmental Microbiology 44, 790-800.

IchikaWa, Y., Kitamoto, Y. \& Hosor, N. (1978). Degradation of polyethylene dodecyl ethers by a pseudomonad isolated from activated sludge. Journal of Fermentation Technology 56, 403-409.

Maggs, J. L., Powell, G. M., Dodgson, K. S., Howes, D., Black, J. G. \& Olavesen, A. H. (1982). Metabolism in the rat of potassium DL-octan-2sulphate, a secondary alkyl sulphate. Xenobiotica 12, 101-109.

Matcham, G. W. J. \& Dodgson, K. S. (1977) Preparation and characterization of substrates suitable for the study of stereospecific secondary alkylsulphohydrolases of detergent-degrading microorganisms. Biochemical Journal 167, 717-722.

Payne, W. J. \& Feisal, V. E. (1963). Bacterial utilization of dodecyl sulphate and dodecyl benzene sulphonate. Applied Microbiology 11, 339-344.

Payne, W. J. \& Painter, B. G. (1971), Resolution by acrylamide gel electrophoresis of alkyl sulphatases and alcohol dehydrogenase. Microbios 3, 199-206.

RATLEDGE, C. (1978). Degradation of aliphatic hydrocarbons. Developments in the Degradation of Hydrocarbons 1, 1-46.

Shore, S. \& Berger, D. R. (1976). Alcohol and ether alcohol sulphates. In Surfactant Science Series. Vol. 7 . Anionic Surfactants, part 1, pp. 135 217. Edited by W. M. Linfield. New York: Marcel Dekker.

Steber, J. \& Wierich, P. (1985). Metabolites and biodegradation pathways of fatty alcohol ethoxylates in microbial biocenoses of sewage treatment plants. Applied and Environmental Microbiology 49, 530-537.

SWISHER, R. D. (1970). Surfactant biodegradation. In Surfactant Science Series, vol. 3, pp. 238-242. New York: Marcel Dekker.

Taylor, A. J., Powell, G. M., Howes, D., Black, J. G. \& Olavesen, A. H. (1978). Metabolism of the surfactants sodium undecyltriethoxy sulphate and sodium dodecyltriethoxy sulphate in the rat. Biochemical Journal 174, 405-412.

VASHON, R. D. \& SCHWAB, B. S. (1982). Mineralization of linear alcohol ethoxylates and linear alcohol ethoxy sulphates at trace concentrations in estuarine waters. Environmental Science and Technology 19 , 433-436.

White, G. F., Russell, N. J. \& DAY, M. J. (1984). A survey of sodium dodecyl sulphate (SDS) resistance and alkylsulphatase production in bacteria from clean and polluted river sites. Encironmental Pollution, Series A 37, 1-11.

Yoshimura, K. \& Masuda, F. (1982). Biodegradation of sodium alkyl poly(oxyalkylene)sulphates. Journal of the American Oil Chemists Society 59, 328-332. 